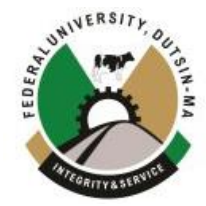

\title{
FORMULATION OF BLOCK SCHEMES WITH LINEAR MULTISTEP METHOD FOR THE APPROXIMATION OF FIRST-ORDER IVPS
}

\author{
*Imoni S. O., Lanlege D. I., Atteh E. M. and Ogbondeminu J. O. \\ Department of Mathematical Sciences, Federal University Lokoja, P.M.B. 1154, Lokoja, Kogi State \\ Corresponding Author’s email: imoni4u@yahoo.com, Sunday.imoni@fulokoja.edu,ng
}

\begin{abstract}
In this paper, formulation of an efficient numerical schemes for the approximation first-order initial value problems (IVPs) of ordinary differential equations (ODE) is presented. The method is a block scheme for some k-step linear multi-step methods ( $k=1,2$ and 3 ) using the Hermite Polynomials a basis function. The continuous and discrete linear multi-step methods (LMM) are formulated through the technique of collocation and interpolation. Numerical examples of ODE have been examined and results obtained show that the proposed scheme can be efficient in solving initial value problems of first order ODE.
\end{abstract}

Keywords: linear multi-step method, ordinary differential equations, initial value problems, Hermite polynomials.

\section{INTRODUCTION}

Numerous problems in Sciences and Engineering are modelled using ordinary differential equations (ODEs). Most of these differential equations do not have analytical solutions which makes numerical methods an option for solving these problems. There are two major discrete variable methods for approximating the solutions of ODEs, namely, one step and linear multi-step methods.

In this paper, we proposed an efficient numerical scheme to solve numerically first order IVPs. The proposed method is a block scheme for some k-step linear multistep methods (for $k=1,2$ and 3 ) using Hermite polynomial as the basis functions. Also, we give the discrete methods used in block and implement it for solving some existing IVPs in the literature. In this paper, we consider the general form of the first order initial value problems.

$$
y^{\prime}(x)=f\left(x, y(x), y\left(x_{0}\right)=y_{0}\right.
$$

Many researchers had developed interest on improving the numerical solution of initial value problems (IVPs) of ordinary differential equation. Consequently, the development of a class of methods called block methods is one of the outcomes. Okunuga and Ehigie (2009) derived two-step continuous and discrete linear multistep methods using power series as a basis function. Akinfenwa et al. (2011) developed a four step continuous block hybrid method with four non-step points for the direct solution of first order initial value problem. Odekunle et al. (2012) developed a continuous linear method using interpolation and collocation for the solution of first-order ODE with constant step size. James et al. (2013), proposed a continuous block method for the solution of second order IVPs with constant step size, the method was developed by interpolation and collocation of power series approximate solution to generate a continuous linear multistep method. A block procedure with linear multistep methods using Legendre polynomials was done by Abualnaja (2015). However, he did not include the block schemes. Okedayo et al. (2018) developed on modified Legendre collocation block method for solving initial value problems of first order ODEs. Also, Okedayo et al. (2018), developed a continuous Laguerre collocation block method for solving initial value problems of first order ordinary differential equations. However, in this paper, Hermite polynomial is used as a basis function to derive some block methods for the solution of first order initial value problem (1). 
FORMULATION OF THE METHODS

We consider the approximate solution of the perturbed form of (1) in the power series below

$$
y_{k}(x)=\sum_{i=0}^{k} c_{i} \psi_{i}(x), \quad x_{n} \leq x \leq x_{n+k}
$$

where

$$
\psi_{i}(x)=x^{i}, \quad i=0,1,2, \ldots, k
$$

Substituting (2) into (1) and add $\lambda H_{k}(x)$ where $\lambda$ is the perturbed term and $H_{k}(x)$ is the Hermite polynomial of degree $k$ valid in $x_{n} \leq x \leq x_{n+k}$ we have

$$
\sum_{i=0}^{k} c_{i} \psi_{i}^{\prime}(x)=f(x, y)+\lambda H_{k}(x)
$$

We shall consider cases where $k=1,2$ and 3 in (2) and (3)

The Hermite polynomial is given by $H i(x), i=0,1,2,3$

$$
H_{0}(x)=1, H_{1}(x)=2 x, H_{2}(x)=4 x^{2}-2, H_{3}(x)=8 x^{2}-12 x
$$

These polynomials are gotten from the Hermite Rodrigue's formula

$$
H_{n}(x)=e^{x^{2}}(-1)^{n} \frac{d^{n}}{d x^{n}}\left(e^{x^{2}}\right)
$$

In this paper, we are going to use the set of polynomials in (5) to formulate the block schemes. Using these polynomials in (5) in the interval $\left[x_{n}, x_{n+k}\right]$, we introduce the change of variable to define the Hermite polynomial as

$$
x=\frac{2 \bar{x}-\left(x_{n+k}+x_{n}\right)}{\left(x_{n+k}-x_{n}\right)}, k=1,2,3
$$

Abualnaja (2015)

\section{For $\mathbf{k}=1$,}

In this case, take $H_{1}(x)=2 x$, since $\mathrm{k}=1$ and use equation (7). Collocate equation (7) at $x_{n}$ and $x_{n+1}$ and solve to obtain

$$
x=\frac{2 \bar{x}-\left(x_{n+k}+x_{n}\right)}{x_{n+k}-x_{n}}=\frac{2 x_{n}-\left(x_{n+1}+x_{n}\right)}{x_{n+1}-x_{n}}=\frac{-x_{n+1}+x_{n}}{x_{n+1}-x_{n}}=-1
$$

Substitute the value of $x$ into $H_{1}(x)=2 x$ and obtain $H_{1}(x)=-2$

Also, following the same procedure for $x_{n+1}$ we have

$$
x=\frac{2 \bar{x}-\left(x_{n+k}+x_{n}\right)}{x_{n+k}-x_{n}}=\frac{2 x_{n+1}-\left(x_{n+1}+x_{n}\right)}{x_{n+1}-x_{n}}=\frac{x_{n+1}+x_{n}}{x_{n+1}-x_{n}}=1
$$

Hence $H_{1}(x)=2$

From equation (2.2), we deduce that $\omega_{0}^{\prime}(x)=0$ and $\omega_{1}^{\prime}(x)=1$. Then substituting into (2.3), we have

$$
f(x, y)=c_{1}-\lambda H_{1}(x)
$$

Thus collocate (10) at $x_{n+1}, i=0,1$ and interpolate (2) at $x=x_{n}$, we obtain a system of three equations with $c_{i},(i=0,1)$ 
and parameter $\lambda$. The system of the equations are given as:

$$
\begin{aligned}
& y_{n}=c_{0}+c_{1} x \\
& f_{n}=c_{1}+2 \lambda \\
& f_{n+1}=c_{1}-2 \lambda
\end{aligned}
$$

Solve (11) to obtain

$$
\lambda=\frac{1}{4}\left(f_{n}-f_{n+1}\right), c_{1}=\frac{1}{2}\left(f_{n}+f_{n+1}\right), c_{0}=y_{n}-\frac{x_{n}}{2}\left(f_{n}+f_{n+1}\right)
$$

From (2) we obtain

$$
\bar{y}=c_{0}+c_{1} x
$$

Now, the required numerical scheme of the proposed method for $y_{n+1}$ will be obtain if we collocate (12) at $x=x_{n+1}$ and substitute the value of $c_{0}, c_{1}$ and $\lambda$. Hence, we obtain

$$
y_{n+1}=y_{n}+\frac{h}{2}\left(f_{n}+f_{n+1}\right)
$$

Now for $k=2$

In this case, $H_{2}(x)=4 x^{2}-2$, since $k=2$ and use equation (7), then collocate the equation at $x_{n}, x_{n+1}$ and $x_{n+2}$ and solve to obtain

$$
x=\frac{2 \bar{x}-\left(x_{n+k}+x_{n}\right)}{x_{n+k}-x_{n}}=\frac{2 x_{n+1}-\left(x_{n+2}+x_{n}\right)}{x_{n+2}-x_{n}}=\frac{-x_{n+2}+x_{n}}{x_{n+2}-x_{n}}=-1
$$

Substitute the value of $\mathrm{x}$ into $H_{2}(x)=4 x^{2}-2$ and obtain Hence $H_{2}(x)=2$

Using the same procedure for $x_{n+1}$ to have

$$
x=\frac{2 \bar{x}-\left(x_{n+k}+x_{n}\right)}{x_{n+k}-x_{n}}=\frac{2 x_{n+1}-\left(x_{n+2}+x_{n}\right)}{x_{n+2}-x_{n}}
$$

Now, put $x_{n+1}$ to be $x_{n}+h$ and $x_{n+2}$ to be equal to $x_{n}+2 h$ in equation (15) to obtain $x=0$. Thus, substitute $\mathrm{x}$ into $H_{2}(x)=4 x^{2}-2$ to get $H_{2}\left(x_{n+1}\right)=-2$

Also for $x_{n+2}$, we have

$$
x=\frac{2 \bar{x}-\left(x_{n+k}+x_{n}\right)}{\left(x_{n+k}-x_{n}\right)}=\frac{2 x_{n+2}-\left(x_{n+2}+x_{n}\right)}{\left(x_{n+2}-x_{n}\right)}=\frac{x_{n+2}-x_{n}}{x_{n+2}-x_{n}}=1
$$

Hence, we substitute the value of $\mathrm{x}$ into $H_{2}(x)=4 x^{2}-2$. Therefore $H_{2}\left(x_{n+2}\right)=2$

From (3), we deduce that $\psi_{0}^{\prime}=0, \psi_{1}^{\prime}=1$ and $\psi_{2}^{\prime}=2 x$. Then equation (4) reduces to the form

$$
f(x, y)=c_{1}+2 x c_{2}-\lambda H_{2}(x)
$$

We now collocate (17) at $x_{n+i}, i=0,1,2$ and interpolate (2) at $x=x_{n}$ to obtain a system of four equations with $c_{i}(i=0,1,2)$ and parameter $\lambda$ 


$$
\begin{aligned}
& y_{n}=c_{0}+c_{1} x_{n}+c_{2} x_{n}^{2} \\
& f_{n}=c_{1}+2 x_{n} c_{2}-2 \lambda \\
& f_{n+1}=c_{1}+c_{2} x_{n+1}+2 \lambda \\
& f_{n+2}=c_{1}+c_{2} x_{n+2}-2 \lambda
\end{aligned}
$$

Solving the system (18) results to

$$
\begin{aligned}
& \lambda=\frac{1}{8}\left(2 f_{n+1}-f_{n+2}-f_{n}\right) \\
& c_{2}=\frac{1}{4 h}\left(f_{n+2}-f_{n}\right) \\
& c_{1}=\frac{1}{4 h}\left(2 h f_{n+1}-2 x_{n} f_{n+2}+2 x_{n} f_{n}-h f_{n+2}+3 h f_{n}\right) \\
& c_{o}=\frac{1}{4 h}\left(4 h y_{n}-2 h x_{n} f_{n+1}+h x_{n} f_{n+2}-3 h x_{n} f_{n}+x^{2} f_{n+2}-x^{2} f_{n}\right)
\end{aligned}
$$

From (2), we obtain

$$
\bar{y}=c_{0}+c_{1} x+c_{2} x^{2}
$$

The required numerical scheme is obtained if we collocate equation (19) at $x=x_{n+1}$ and substitute $c_{0}, c_{1}, c_{2}$ and $\lambda$ as

$$
y_{n+1}=y_{n}+\frac{h}{4}\left(f_{n+2}+2 f_{n+1}+f_{n}\right)
$$

\section{Now consider $k=3$}

In this case, take $H_{2}(x)=8 x^{3}-12 x$, since $k=3$ and use equation (7). Collocate this equation at $x_{n}, x_{n+1}, x_{n+2}$ and $x_{n+3}$ and solve to have

$$
x=\frac{2 \bar{x}-\left(x_{n+k}+x_{n}\right)}{\left(x_{n+k}-x_{n}\right)}=\frac{2 x_{n}-\left(x_{n+3}+x_{n}\right)}{x_{n+3}-x_{n}}=\frac{-x_{n+3}+x_{n}}{x_{n+3}-x_{n}}=-1
$$

Thus substitute the value of $x$ into $H_{3}(x)=8 x^{3}-12 x$ and obtain $H_{3}(x)=4$

Following the same process for $x_{n+1}$ we have

$$
x=\frac{2 x_{n+1}-\left(x_{n+3}+x_{n}\right)}{x_{n+3}-x_{n}}
$$

Put $x_{n+1}=x_{n}+h, x_{n+3}=x_{n}+3 h$ and obtain

$$
\frac{2\left(x_{n}+h\right)-\left(x_{n}+3 h+x_{n}\right)}{x_{n}+3 h-x_{n}}=-\frac{1}{3} \text {. }
$$

By substituting the value of $x$ into $H_{3}(x)=8 x^{3}-12 x$, then $H_{3}(x)=\frac{100}{27}$

Follow the same procedure for $x_{n+2}$ and have

$x=\frac{2 x_{n+2}-\left(x_{n+3}+x_{n}\right)}{x_{n+3}-x_{n}}$. Putting $x_{n+2}=x_{n}+2 h, x_{n+3}=x_{n}+3 h$ and substituting into the above equation to obtain 


$$
\frac{2\left(x_{n}+2 h\right)-\left(x_{n}+3 h+x_{n}\right)}{x_{n}+3 h-x_{n}}=\frac{1}{3} \text {. Substituting the value of } x \text { into } H_{3}(x)=8 x^{3}-12 x \text {, then } H_{3}(x)=-\frac{100}{27}
$$

Following the same procedure for $x_{n+3}$, we have

$$
x=\frac{2 \bar{x}-\left(x_{n+k}+x_{n}\right)}{\left(x_{n+k}-x_{n}\right)}=\frac{2 x_{n+3}-\left(x_{n+3}+x_{n}\right)}{x_{n+3}-x_{n}}=1
$$

Thus, substitute the value of $x$ into $H_{3}(x)=8 x^{3}-12 x$, and have $H_{3}(x)=-4$

Thus, from equation (3), we deduce that $\psi_{0}^{\prime}(x)=0, \psi_{1}^{\prime}(x)=1, \psi_{2}^{\prime}(x)=2 x, \psi_{3}^{\prime}(x)=3 x^{2}$.

Equation (3) reduces to the form

$$
f(x, y)=c_{1}+2 x c_{2}+3 x^{2} c_{3}-\lambda H_{3}(x)
$$

We now collocate (23) at $x_{n+i}(i=0,1,2)$ and interpolate (2) at $x=x_{n}$ to obtain a system of five equations with $c_{i}(i=0,1,2,3)$ and parameter $\lambda$ as

$$
\begin{aligned}
& y_{n}=c_{0}+c_{1} x_{n}+c_{2} x_{n}^{2}+c_{3} x_{n}^{3} \\
& f_{n}=c_{1}+2 c_{2} x_{n}+3 c_{3} x_{n}^{2}-4 \lambda \\
& f_{n+1}=c_{1}+2 c_{2} x_{n+1}+3 c_{3} x_{n+1}^{2}-\frac{100}{27} \lambda \\
& f_{n+2}=c_{1}+2 c_{2} x_{n+2}+3 c_{3} x_{n+2}^{2}+\frac{100}{27} \lambda \\
& f_{n+3}=c_{1}+2 c_{2} x_{n+3}+3 c_{3} x_{n+3}^{2}+4 \lambda
\end{aligned}
$$

Solving the system (24) resulted to

$$
\begin{aligned}
\lambda= & \frac{9}{128}\left(f_{n}-3 f_{n+1}+3 f_{n+2}-f_{n+3}\right) \\
c_{3}= & \frac{1}{12 h^{2}}\left(f_{n}-f_{n+1}-f_{n+2}+f_{n+3}\right) \\
c_{2}= & -\frac{1}{96 h^{2}}\left(61 h f_{n}-63 h f_{n+1}-9 h f_{n+2}+11 h f_{n}+3+24 f_{n} x_{n}-24 f_{n+1} x_{n}-29 f_{n}+2 x_{n}+24 f_{n} x_{n}\right) \\
c_{1}= & -\frac{1}{96 h^{2}}\left(123 h^{2} f_{n}-81 h^{2} f_{n+1}+81 h^{2} f_{n+2}-27 h^{2} f_{n+3}+122 h f_{n} x_{n}-126 h f_{n+1} x_{n}-\right. \\
& \left.18 h f_{n+2} x_{n}+22 f_{n+3} x_{n}+24 f_{n} x_{n}^{2}-24 f_{n+1} x_{n}^{2}-24 f_{n+2} x_{n}^{2}+24 f n+3 x_{n}^{2}\right) \\
c_{0}= & -\frac{1}{96 h^{2}}\left(123 h^{2} f_{n} x_{n}-81 h^{2} f_{n+1} x_{n}+81 h^{2} f_{n+2} x_{n}-27 h^{2} f_{n+3} x_{n}+61 h f_{n} x_{n}^{2}-\right. \\
& 63 h f_{n+1} x_{n}^{2}-9 h f_{n+2} x_{n}^{2}+11 h f_{n+3} x_{n}^{2}+8 f_{n} x_{n}^{3}-8 f_{n+1} x_{n}^{3}-8 f_{n+2} x_{n}^{3}+8 f_{n+3} x_{n}^{3}- \\
& 96 h^{2} y_{n}
\end{aligned}
$$

Again, from (2) we have

$$
\bar{y}=c_{0}+c_{1} x+c_{2} x^{2}+c_{3} x^{3}
$$

The required numerical scheme is then obtained if we collocate (25) at $x_{n+1}$ and substituting for $c_{0}, c_{1}, c_{2}$ and $c_{3}$ as 


$$
y_{n+1}=y_{n}+\frac{h}{48}\left(35 f_{n}-13 f_{n+1}+41 f_{n+2}-15 f_{n+3}\right)
$$

Now we formulate the block schemes for the polynomials of cases $k=1,2$ and 3

For $k=1$, collocate equation (12) at $x=x_{n+1}, x_{n+2}, x_{n+3}$ to obtain

$$
\begin{aligned}
& y_{n+1}=y_{n}+\frac{h}{2}\left(f_{n}+f_{n+1}\right) \\
& y_{n+2}=y_{n}+h\left(f_{n}+f_{n+1}\right) \\
& y_{n+3}=y_{n}+\frac{3 h}{2}\left(f_{n}+f_{n+1}\right)
\end{aligned}
$$

For $k=2$, collocate equation (20) at $x=x_{n+1}, x_{n+2}, x_{n+3}$ to obtain

$$
\begin{aligned}
& y_{n+1}=y_{n}+\frac{h}{4}\left(f_{n}+2 f_{n+1}+f_{n+2}\right) \\
& y_{n+2}=y_{n}+\frac{h}{2}\left(f_{n}+2 f_{n+1}+f_{n+2}\right) \\
& y_{n+3}=y_{n}+\frac{3 h}{4}\left(6 f_{n}+2 f_{n+1}+f_{n+2}\right)
\end{aligned}
$$

For $k=3$, collocate equation (25) at $x=x_{n+1}, x_{n+2}, x_{n+3}$ to obtain

$$
\begin{aligned}
& y_{n+1}=y_{n}+\frac{h}{48}\left(35 f_{n}-13 f_{n+1}+41 f_{n+2}-15 f_{n+3}\right) \\
& y_{n+2}=y_{n}+\frac{h}{48}\left(33 f_{n}+13 f_{n+1}+67 f_{n+2}-17 f_{n+3}\right) \\
& y_{n+3}=y_{n}+\frac{3 h}{8}\left(f_{n}+3 f_{n+1}+3 f_{n+2}+f_{n+3}\right)
\end{aligned}
$$

\section{ERORR ANALYSIS OF THE METHOD}

In this section, we discuss the order, the error constant and convergence of the proposed block schemes. The proposed schemes in this paper belong to the class of linear multi-step method (LMM) which is of the form

$$
\sum_{j=0}^{k} \alpha_{j}(x) y\left(x_{n+j}\right)=h \sum_{j=0}^{k} \beta_{j}(x) f\left(x_{n+j}\right)
$$

According to Sastry (2008) and Lambert (1981), define the order and error constant associated with (30) to be the linear difference operator

$$
\ell[y(x) ; h]=\sum_{j=0}^{k}\left\{\alpha_{j} y(x+j h)-h \beta_{j} y^{\prime}(x+j h)\right\}
$$

Assuming that $y(x)$ is continuously differentiable on the interval $[a, b]$, we can expand the terms in (31) as a Taylor series about the point $x$ to obtain the expansion

$$
\ell[y(x) ; h]=c_{0} y(x)+c_{1} h y^{\prime}(x)+c_{2} h^{2} y^{\prime \prime}(x)+\ldots+c_{q} h^{q} y^{q}(x)
$$

where the constant coefficients $c_{q}, q=0,1, \ldots$ are given as follows:

$$
c_{0}=\sum_{j=0}^{k} \alpha_{j}, c_{1}=\sum_{j=0}^{k}\left(j \alpha_{j}-\beta_{j}\right), \ldots, c_{q}=\sum_{j=0}^{k}\left[\frac{1}{q !} j^{q} \alpha_{j}-\frac{1}{(q-1) !} j^{q-1} \beta_{j}\right], q=2,3 \ldots
$$


We say that the methods are said to be order $\mathrm{p}$ if $c_{0}=c_{1}=c_{2}=\ldots=c_{p}=0, c_{p+1} \neq 0$

Therefore the $c_{p+1}$ is the error constant.

Hence, we establish that (27), (28) and (29) are of the following orders and error constants respectively.

when $k=1, p=1$ and $c_{p+1}=\frac{1}{4}$

when $k=2, p=2$ and $c_{p+1}=-\frac{1}{6}$

when $k=3, p=4$ and $c_{p+1}=-\frac{864}{23040}$

\section{NUMERICAL EXAMPLES}

In this section, two initial value problems were solved using the proposed method for $k=2$ and $k=3$ and compare the numerical results obtained with the exact solution and those obtained from using the method of Okedayo et al (2018) in order to test the efficiency of the schemes.

\section{Example 1}

Consider the following IVP

$$
y^{\prime}(x)=-y, y(0)=1
$$

with the exact solution $y(x)=e^{-x}$

\section{Example 2}

Consider the IVP

$y^{\prime}(x)=x-x y, y(0)=0$
With exact solution $y(x)=1-e^{-\frac{x^{2}}{2}}$

Notations: BLS $=$ Block schemes derived in this paper, $\mathrm{LM}=$ Method of Okedayo et al. (2018), Exact = Exact Solution, $\mid$ Exact-BLS $\mid=$ the absolute value of the exact solution minus computed solution of the method derived in this paper and $\mid$ Exact-LM $\mid=$ the absolute value of the exact solution minus computed solution of Okedayo et al. (2018).

The numerical results of these examples are depicted in Tables 1,23 and 4 with $k=2$ and $k=3$ with constant step size of $h=0.1$ respectively. In tables 1 and 3 we presented a comparison of the obtained numerical results using the proposed scheme with the exact solution and Table 2 and 4 presents the comparison of the results obtained from proposed scheme, the exact solution and those numerical results obtained from Okedayo et al. (2018).

Table 1: A comparison of numerical results of proposed Scheme at $k=2$ with exact solution for Example 1

\begin{tabular}{|l|l|l|l|}
\hline x-value & $\begin{array}{l}\text { BLS } \\
k=2\end{array}$ & Exact & $\mid$ Exact-BLS $\mid$ \\
\hline 0.0 & 1.000000 & 1.000000 & 0.000000 \\
\hline 0.1 & 0.905090 & 0.904837 & $4.253 \times 10^{-4}$ \\
\hline 0.2 & 0.818181 & 0.818730 & $5.49 \times 10^{-4}$ \\
\hline 0.3 & 0.749092 & 0.740818 & $8.274 \times 10^{-3}$ \\
\hline 0.4 & 0.675227 & 0.670320 & $4.907 \times 10^{-3}$ \\
\hline $\mathrm{i} 0.5$ & 0.608761 & 0.606531 & $2.23 \times 10^{-3}$ \\
\hline 0.6 & 0.548760 & 0.548811 & $5.1 \times 10^{-3}$ \\
\hline 0.7 & 0.504028 & 0.496585 & $7.443 \times 10^{-3}$ \\
\hline 0.8 & 0.448139 & 0.449328 & $1.189 \times 10^{-3}$ \\
\hline 0.9 & 0.416232 & 0.406569 & $9.663 \times 10^{-3}$ \\
\hline 1.0 & 0.373893 & 0.367879 & $6.014 \times 10^{-3}$ \\
\hline
\end{tabular}


Table 2: A comparison of numerical results of proposed scheme at $k=3$ with exact solution and LM for Example 1

\begin{tabular}{|l|l|l|l|l|l|}
\hline x-value & $\begin{array}{l}\text { BLS } \\
k=3\end{array}$ & LM & Exact & $\mid$ Exact-BLS $\mid$ & $\mid$ Exact-LM $\mid$ \\
\hline 0.0 & 1.000000 & 1.000000 & 1.000000 & 0.000000 & 0.000000 \\
\hline 0.1 & 0.904808 & 0.905953 & 0.904837 & $2.9 \times 10^{-5}$ & $1.116 \times 10^{-3}$ \\
\hline 0.2 & 0.818705 & 0.820856 & 0.818730 & $5.0 \times 10^{-} 5$ & $2.126 \times 10^{-3}$ \\
\hline 0.3 & 0.740823 & 0.743857 & 0.740818 & $5.0 \times 10^{-5}$ & $3.039 \times 10^{-3}$ \\
\hline 0.4 & 0.670304 & 0.674185 & 0.670320 & $1.6 \times 10^{-5}$ & $3.865 \times 10^{-3}$ \\
\hline 0.5 & 0.606516 & 0.611143 & 0.606531 & $1.5 \times 10^{-5}$ & $4.612 \times 10^{-3}$ \\
\hline 0.6 & 0.548820 & 0.554100 & 0.548811 & $9.0 \times 10^{-6}$ & $5.289 \times 10^{-3}$ \\
\hline 0.7 & 0.496576 & 0.502486 & 0.496585 & $9.0 \times 10^{-6}$ & $5.901 \times 10^{-3}$ \\
\hline 0.8 & 0.449321 & 0.455784 & 0.449328 & $7.0 \times 10^{-6}$ & $6.456 \times 10^{-3}$ \\
\hline 0.9 & 0.406578 & 0.413527 & 0.406569 & $9.0 \times 10^{-6}$ & $6.958 \times 10^{-3}$ \\
\hline 1.0 & 0.367876 & 0.375290 & 0.367879 & $3.0 \times 10^{-6}$ & $7.411 \times 10^{-3}$ \\
\hline
\end{tabular}

Table 3: A comparison of numerical results of proposed scheme at $k=2$ with exact solution for Example 2

\begin{tabular}{|l|l|l|l|}
\hline $\mathrm{x}$-value & $\begin{array}{l}\text { BLS } \\
k=2\end{array}$ & Exact & $\mid$ Exact-BLS $\mid$ \\
\hline 0.0 & 0.000000 & 0.000000 & 0.000000 \\
\hline 0.1 & 0.005452 & 0.004785 & $6.67 \times 10^{-4}$ \\
\hline 0.2 & 0.018904 & 0.018901 & $1.97 \times 10^{-4}$ \\
\hline 0.3 & 0.042261 & 0.033013 & $9.218 \times 10^{-3}$ \\
\hline 0.4 & 0.058942 & 0.056862 & $2.078 \times 10^{-3}$ \\
\hline 0.5 & 0.117427 & 0.115602 & $1.825 \times 10^{-3}$ \\
\hline 0.6 & 0.121342 & 0.166729 & $4.5387 \times 10^{-2}$ \\
\hline 0.7 & 0.178557 & 0.216929 & $1.1828 \times 10^{-2}$ \\
\hline 0.8 & 0.235772 & 0.255531 & $1.9758 \times 10^{-2}$ \\
\hline 0.9 & 0.274301 & 0.292022 & $1.37121 \times 10^{-2}$ \\
\hline 1.0 & 0.327572 & 0.353368 & $2.5706 \times 10^{-2}$ \\
\hline
\end{tabular}


Table 4: A comparison of numerical results of proposed scheme at $k=3$ with exact solution and LM for Example 2

\begin{tabular}{|l|l|l|l|l|l|}
\hline $\mathrm{x}$-value & $\begin{array}{l}\text { BLS } \\
k=3\end{array}$ & LM & Exact & $\mid$ Exact-BLS $\mid$ & $\mid$ Exact-LM $\mid$ \\
\hline 0.0 & 0.000000 & 0.000000 & 0.000000 & 0.000000 & 0.000000 \\
\hline 0.1 & 0.005073 & 0.005892 & 0.004785 & $2.88 \times 10^{-5}$ & $1.027 \times 10^{-4}$ \\
\hline 0.2 & 0.018214 & 0.020465 & 0.018901 & $6.87 \times 10^{-4}$ & $1.564 \times 10^{-3}$ \\
\hline 0.3 & 0.039568 & 0.037475 & 0.033013 & $6.555 \times 10^{-3}$ & $4.402 \times 10^{-3}$ \\
\hline 0.4 & 0.064584 & 0.078865 & 0.056862 & $7.222 \times 10^{-3}$ & $2.2003 \times 10^{-3}$ \\
\hline 0.5 & 0.107592 & 0.120033 & 0.115602 & $8.01 \times 10^{-3}$ & $4.4431 \times 10^{-3}$ \\
\hline 0.6 & 0.161899 & 0.159353 & 0.166729 & $4.83 \times 10^{-3}$ & $7.376 \times 10^{-3}$ \\
\hline 0.7 & 0.212809 & 0.221055 & 0.216929 & $4.1214 \times 10^{-3}$ & $2.362 \times 10^{-2}$ \\
\hline 0.8 & 0.250814 & 0.279245 & 0.255531 & $2.4716 \times 10^{-2}$ & $2.3715 \times 10^{-2}$ \\
\hline 0.9 & 0.308725 & 0.328348 & 0.292022 & $1.6703 \times 10^{-2}$ & $3.6326 \times 10^{-2}$ \\
\hline 1.0 & 0.384329 & 0.398478 & 0.353368 & $3.0961 \times 10^{-2}$ & $1.35202 \times 10^{-1}$ \\
\hline
\end{tabular}

Tables 1, 2, 3 and 4 shows that the proposed schemes approximate the solutions of initial value problems given in Examples 1 and 2 as the absolute errors are convergent. Also, the absolute errors presented in Tables 2 and 4 show that the proposed schemes compared favourably with the method of Okedayo et al. (2015) applied to the given numerical examples.

\section{CONCLUSION}

In this research work, a class of three new block schemes for the approximation of initial value problems of first order ordinary differential equations using Hermite polynomial as a basis function has been obtained. The proposed method was used to solve numerically some initial value problems and the results compared with the exact solutions and the method of Okedayo et al. (2015). From the numerical results, it is observed that the new schemes were capable for solving first order IVPs as generated results compared favorably with the existing method and the exact solutions. The method is very simple to implement.

\section{REFERENCES}

Abualnaja K. M. (2015). A block procedure with linear multistep methods using Legendre polynomials for solving ODEs, Applied Mathematics, 6, 717-723

Abubakar M.B., Ali B. and Muktar I.B (2012). Formulation of predictor-corrector methods from 2-step hybrid Adams methods for the solution of initial value problems of ordinary differential equations, International Journal of Engineering and Science, vol. 5 , No. 3

Adegboye Z.A and Aliyu I. (2012). A 4-step implicit collocation method for solution of first and second order odes, International Journal of Science and Technology, vol. 1 No. 11, 644-649

Akinfenwa O.A, Yao N.M and Jator S.N (2011). A linear multistep hybrid method with continuous coefficient for solving stiff odes, Journal of Mordern Mathematics and Statistics, vol. 5(2), 47-5
Ehigie J.O., Okunuga S.A, Sofoluwe A.B and Akanbi M.A (2010). Generalized two-step continuous linear multistep method of hybrid type for the integration of second order ODEs, Achieves of Applied Science and Research, vol.2 No.6, 362-372

Fatokun J.O., Onumanyi P. and Sirisena U. (1999). A multistep collocation based on exponential basis for stiff initial value problems, Nigerian journal of Mathematics and Applications, vol.12, 207-223

Fatunla S.O. (1998). A numerical method for initial value problems in ODEs, Academic Press Inc, New york

James A.A., Adesanya A.O., Odekunle M.R. and Yakubu D.G. (2013).Constant order predictor corrector method for the solution of modeled problems of first order initial value problems of odes, intewrnational journal of Mathematical computational, statistics, natural and Physical Engineering, vol. 7 , No. 11

Lambert J.D. (1991).Numerical methods for ODE systems, John Wiley,Chichesster

Odekunle M.R., Adesanya A.O. and Sunday J. (2012). A new block integrator for the solution of initial vlue problems of first order odes, International Journal of Pure and Applied Sciences and Technology, 11(1), 92-100

Okedayo T. G., Owolanke A.O., Amumeji O.T., Owolanke A.O. and Ogunbamike O.K. (2018). Modified Legendre collocation block method for solving initial value problems of first order odes, open access library journal, 5: e4565, https://doi.org/10.4236/oalib.1104565 
Okedayo T. G., Amumeji O.T and Adesuyi M.P. (2018). Sastry S.S. (2008). Introductory methods of Numerical Modified Laguerre collocation block method for solving initial Analysis, Prentice-Hall of India Private Limited, New Delhivalue problems of first order odes, Journal of Advances in 110001

Mathematics and Computer Science, 29 (2), 1-13

(C)2020 This is an Open Access article distributed under the terms of the Creative Commons Attribution 4.0 International license viewed via https://creativecommons.org/licenses/by/4.0/ which permits unrestricted use, distribution, and reproduction in any medium, provided the original work is cited 